Dr. G. A. Friedman, who made a diagnosis of acquired hemolytic jaundice and advised splenectomy. Except for diseases of childhood, the nature of which he did not know, the patient had had no previous illness. He had come to America from Italy eighteen months before. Jaundice was first noted about nine months before he came to the hospital, and had been persistent up to the time of admission.

Examination showed a rather slender, well-nourished young man, with marked general icterus of a lemon yellow hue. His spleen extended to within an inch of the umbilical level in the mammary line.

The urine contained urobilin and urobilinogen; the feces were of normal color and contained bile and also urobilin.

The blood examination showed a moderate degree of secondary anemia; 78 per cent. hemoglobin; 4,200,000 red cells, with 16 per cent. postvital reticulation, and a normal differential count. Auto-agglutination tests were negative. Two fragility tests before operation showed a minimum resistance of 0.48 to 0.50 per cent., and a maximum resistance of 0.30 to 0.35 per cent.

Splenectomy was performed on November 23, 1915, at the Roosevelt Hospital. The gallbladder and ducts, liver, stomach and pancreas were normal. The spleen measured 17 by 10 by $7 \mathrm{~cm}$. and weighed $800 \mathrm{gm}$. It was slightly adherent along the posterior border and at the upper pole, but delivered without great difficulty and the operation was well borne.

The jaundice began to fade within two or three days and was entirely gone by the tenth day. The patient has remained well and entirely free from jaundice since the operation.

The spleen was rather soft and flabby, the cut surface was dark red and rather granular, and the trabeculations stood out prominently. Microscopically, there was no increase of fibrous tissue; the follicles were of varying size, some having disappeared. There was marked interstitial suffusion with red blood cells. The changes in the pathology of the spleens removed have been fairly uniform and correspond with those found in previous reports. There is marked interstitial suffusion of red blood cells, dilatation of splenic sinuses, loss of follicles and absence of germinal centers. There is practically no increase of fibrous tissue.

Six days after splenectomy the fragility test showed a minimum resistance of 0.50 per cent. and a maximum of 0.35 per cent., that is, there was no change following the operation. On December 20, four weeks after operation, the fragility test was the same, reticulation of the red cells was normal ( 0.5 per cent.), and urobilin and urobilinogen had disappeared from the urine. The patient gained in weight and resumed his work four weeks after the operation.

This case illustrates the acquired type of hemolytic jaundice, evidently dependent on the splenic changes and on no previous etiologic factor that could be determined.

The fragility of the red blood cells in this case was slight, and was practically unchanged after splenectomy.

The third case was of the congenital, nonfamilial type, and the patient was operated on by my colleague, Dr. James I. Russell, to whom I am indebted for permission to include it in this report. Only a brief abstract of the history will be given, as the case was presented to the New York Surgical Society and reported in its Procecdings.

The patient was a man, 20 years of age, transferred from the medical service to the second surgical division on July $6,1915 \cdot$ (history No. 6546).

He had been jaundiced since birth, the icterus increasing up to 5 years of age, after which time it was stationary. There was no history of jaundice in the family. He presented the following symptoms: Icterus of a fairly marked degree, nonobstructive in type, of a lemon yellow hue, secondary anemia of a mild type ; 74 per cent. hemoglobin, $5,200,000$ red cells; red cell fragility not increased, the minimum resistance being 0.45 per cent., and the maximum 0.30 per cent., remaining unchanged after operation; reticulated red cells, vital stain, 8 per cent.; splenomegaly of a marked degree, the spleen weighing $1,143 \mathrm{gm}$.

Splenectomy was performed on July 10, 1915, and was followed by complete disappearance of the jaundice and a prompt recovery.

In addition to the three cases cited, I have recently performed cholecystectomy for calculi with chronic cholecystitis in a typical case of congenital hemolytic jaundice of the familial type, with marked splenomegaly. Both the patient and her physician were unwilling to have splenectomy performed at that time, though recognizing the possible necessity of such a procedure in the future.

That hemolytic jaundice is not uncommon and that it can be cured by splenectomy are now well recog-; nized, and recent contributions to the literature are emphasizing its importance and bringing it more and more to the attention of the profession.

\section{INDICATIONS FOR SPLENECTOMY IN CERTAIN CHRONIC BLOOD DISORDERS}

\author{
THE TECHNIC OF THE OPERATION * \\ DONALD C. BALFOUR, M.D. \\ ROCHESTER, MINN.
}

Splenectomy has already proved a curative measure in certain diseases associated with definite blood changes, and the operation has recently been advocated for other diseases which appear to more or less closely related to those in which the spleen is now the known causative factor. The basis on which splenectomy has been suggested in these heretofore incurable conditions will be more clearly understood if we briefly review the development which has resulted in the present situation.

Splenic anemia offers the most familiar example of the therapeutic value of splenectomy. Sufficient time has now elapsed since the earlier operations for this disease to prove that the results of splenectomy in early or in moderately advanced cases are not only excellent, but permanent. The most interesting characteristic of the disease as concerns the purpose of this paper is its tendency to develop cirrhotic changes in the liver. In fact, Banti described the disease as it appeared in its later stages when it is associated with marked cirrhosis and with ascites. In such a stage, from a diagnostic standpoint, it is often difficult and sometimes impossible to determine in a given case whether the condition is the result of a true splenic anemia or of a primary liver cirrhosis associated with a large spleen. Experience has shown that in the very late stages of splenic anemia definite improvement following splenectomy cannot be expected, yet it is also true that a moderate cirrhosis, even associated with ascites, has been present in some cases in which excellent results have been obtained. This fact, therefore, with the probability that certain of the cases which have been classified as true splenic anemias and operation performed on that diagnosis, were in reality cases of primary cirrhosis, was responsible for the sugges-

* Read before the joint meeting of the Section on Practice of Medicine and the Section on Surgery, General and Abdominal, at the Sixty.Seventh Annual Session of the American Medical Association, Detroit, June, 1916. 
tion that splenectomy might prove of value in arresting the progress of selected types of hepatic cirrhosis.

The interdependence of spleen and liver is not clearly understood, yet there is ample evidence to show that some of the most important functions of the liver, for example, its hemolytic power, are more or less controlled by the spleen. Certain pathologic conditions in the liver, therefore, are the result of overactivity, which may in turn be due to overstimulation by the spleen.

During the last two years, with this theory as a basis, selected patients with primary hepatic cirrhosis have been splenectomized. Two, at least, of four such cases of our own gave promise that the removal of the spleen might stop or retard the progress of the disease, but we are not yet in a position to state the value of such a procedure.

The success of splenectomy in splenic anemia, with associated splenomegaly and severe anemia, has led to splenectomy in other diseases in which splenomegaly and anemia are characteristics. One of the most interesting of these is the syphilitic spleen; we have seen good results follow splenectomy in three cases of this form of syphilis. The prominent clinical features of the cases in which we believed operation to be indicated were (1) splenomegaly, (2) a history of syphilis not responding to treatment, (3) positive Wassermanns, (4) anemia. It is important to note that previous to operation a negative Wassermann could be obtained under appropriate treatment, but the reaction became positive as soon as treatment was discortinued. All these patients had gummas in the liver, and in the splcens removed the spirochete could be demonstrated in large numbers. It was also a clinical observation that although the virulence of the infection could be more or less controlled by antisyphilitic treatment, it was not eradicated.

From the foregoing facts, the conclusion may be drawn that the spleen picks up the spirochetes and harbors them, and this conclusion is supported by the surgical observation that removal of the spleen, followed by antisyphilitic treatment, results in prompt and permanent relief.

The influence which the spleen exercises on the hemolytic function of the liver and spleen is most strikingly illustrated in the effect of splenectomy in hemolytic jaundice. Although this disease is a problem from an etiologic standpoint, the most important therapeutic fact is that splenectomy is followed by prompt, complete and permanent cure. The rapid disappearance of the icterus, which is the most obvious symptom of the disease, is an amazing clinical result; the anemia, which is sometimes severe, is also rapidly corrected.

The remarkable results following splenectomy in splenic anemia and hemolytic jaundice, particularly the latter, appear to have provided the basis on which Eppinger and others have suggested that similar surgical treatment might prove of value in pernicious anemia. It is important, therefore, that the relationship of splenectomy to these other diseases should be borne in mind. The argument becomes more forceful if it is possible to establish similarities between any one of these diseases for which splenectomy is curative, and pernicious anemia. Many of the features of hemolytic jaundice appear to have a counterpart in pernicious anemia, and, in fact, Eppinger believes that the chief, and possibly the only, difference between hemolytic jaundice and pernicious anemia is that in the former the bone marrow is able to compensate for the increased blood destruction, whereas in pernicious anemia it soon loses this power, and the disease eventually is dependent on a bone marrow disability.

It would, therefore, appear as a logical sequence that since splenectomy has proved a specific in hemolytic jatundice, it would sooner or later be advocated in pernicious anemia. Although the first operations for this disease were performed, both abroad and in this country, about the same time (1913), to Eppinger must be given special credit for efforts to establish a scientific basis for the surgical treatment. While the surgical therapeusis of pernicious anemia can be considered as on trial, it presents features of much interest and promise, and splenectomy is now being performed with a frequency which should soon permit definite and decisive deductions.

It must, of course, be primarily recognized that any new therapeusis in a disease of the character of pernicjous anemia must be subjected to a particularly thorough trial. The extreme chronicity of the disease in its usual course, and the fact that, with or without treatment, remissions occur, during which the patient is much improved and the blood may even revert to normal, stamps any conclusions based on the early results of operation as premature. This much, however, can be said, that immediate improvement follows splenectomy more consistently and promptly than any treatment heretofore used.

It is on the ultimate results, however, which are as yet insufficient, that the final decision as to the real effciency of splenectomy in pernicious anemia must rest. There is at the present time, therefore, decided uncertainty as to any superior therapeutic value of a permanent character possessed by splenectomy. It is also true that pernicious anemia has thus far been an incurable disease, ranking in fatality with cancer and less amenable to successful treatment. The certainty of the ultimate prognosis has been the outstanding feature of our knowledge of the disease, and the doomed patient could hope only that the disease would be slowly progressive and not associated with great physical disability.

The pathologic picture which Eppinger has described as distinctive of the spleen in pernicious anemia is as follows: The pulp areas in the spleen, and only these, are gorged with blood. Eppinger explains this engorgement as a direct result of an abnormal condition of the central artery. It has been shown that the entire wall of this artery is greatly thickened, and that the greatest change takes place in the media. The theory is, briefly, that sufficient obstruction results to force the flow of blood through the capillaries into the pulp areas, where the erythrocytes are destroyed. If a constant pathology can be demonstrated in the spleen in pernicious anemia, it will be a great advance toward the rational treatment of the disease.

While it cannot be held that the basis on which Eppinger and his followers have endeavored to establish the surgical treatment of the disease has been confirmed or even well supported by others, it is not essential, nor is it good judgment, to postpone surgical treatment of the disease until an acceptable basis for such treatment is generally recognized.

The hemolytic nature of pernicious anemia is demonstrated by the increased amounts of urobilin and urobilinogen excreted. It has been assumed, and there is much clinical and experimental evidence to show, 
that this hyperhemolysis is due to a hypersplenism, and interesting investigations are now being conducted, after the suggestion of Schneider, to determine the exact quantity of these hemolytic products by obtaining them immediately with a duodenal tube on their exit from the liver. Giffin has shown, by the records of the urobilin and urobilinogen estimation in a series of cases of pernicious anemia, that the values appeared to run more or less parallel to the degree of icterus, and that in some cases at least there was a marked fall in the value of the blood-derived pigments after splenectomy. It is possible that from this method information of at least prognostic value may be derived. Giffin has suggested the very interesting possibility that the relation of hemolysis, as shown by the estimation of the blood-derived pigments, and the bone marrow disability, as illustrated by the blood picture, may result in deductions of decided value.

The effect of splenectomy in other diseases being the primary basis for the operation in pernicious anemia, the further indications must be mainly based on operative results, and as early results only are as yet available, such indications are presented with reservation and will undoubtedly be subject to further modification.

\section{INDICATIONS FOR OPERATION}

Size of Spleen.-It would appear that the larger the spleen the better the prospects that splenectomy will prove of benefit. It may be assumed in this disease that oversize means overactivity, and it has certainly been true, in our own experience, that the most consistently promising results following splenectomy have occurred when the spleen showed the greatest enlargement. However, good immediate results have been obtained also when little or no enlargement was present. The palpability of the spleen, therefore, has been looked on as one of the first considerations in discussing the advisability of splenectomy. Exclusive of three cases in which the spleens were very large, the spleens in a series of thirty-seven of our cases averaged $400 \mathrm{gm}$., as compared with the normal of about $195 \mathrm{gm}$. It should be remembered that any estimation of the size of the spleen before it has become actually palpable is rather speculative. W. J. Mayo early pointed out that frequently in exploring the spleen in the course of abdominal operations, and comparing its actual size with that noted in the clinical records, it is found that clinical records are not consistently correct and as a rule are accurate only when the organ is palpable.

Icteric Types of the Disease.-The very intimate relationship between the spleen and the acholuric icterus as seen in pernicious anemia, hemolytic jaundice and in hypertrophic cirrhosis is proved both by operative results and experimental studies. The remarkably rapid, complete and permanent disappearance of the jaundice in hemolytic jaundice, for example, following splenectomy is sufficient evidence of the splenic factor. Experimentally, it is very difficult to produce toxic jaundice in spleenless dogs by those poisons which readily produce it in the normal dog. In spleenless dogs under the administration of such poisons (particularly toluylendiamin) the bile remains thin and green, while in nonsplenectomized dogs it becomes viscid. Stadelmann was the first to attribute such acholuric jaundice to this thickening of the bile, which delays, by very reason of its viscosity, its own passage through the finer bile capillaries. A marked icteric discoloration in the skin in pernicious anemia may be taken as an indication that the spleen is to a large degree responsible for the abnormal blood destruction feature in the disease.

Response to Transfusion.-The transfusion of blood, particularly of small quantities at frequent intervals, has for some time been recognized as valuable treatment, though only of temporary character, in pernicious anemia. It has recently become evident, however, that transfusion is not only an excellent therapeutic expedient, but that in the immediate response following the procedure, it is of distinct prognostic value in foretelling the probable results of splenectomy. Definite improvement after transfusion is a strong indication for splenectomy, but when no benefit occurs from repeated transfusions, splenectomy will probably also prove futile, though the reverse of both these indications is occasionally true. Transfusion seems to serve a double purpose, as does the ligation of the blood supply in exophthalmic goiter, by (1) improving the condition of the patient, and by (2) predicting rather accurately the effect of the major operation on the disease.

Duration of the Disease.-Although in our own experience satisfactory results may be obtained when the disease has been of long standing, we believe the results are not as favorable as those in cases in which the disease has existed a short time.

Radical measures to interrupt the progress of the disease while it is yet in an early stage seem logical. Although there is little positive knowledge as to the reason for the permanent disability of the bone marrow, it is probable that it is constantly overstimulated in an effort to meet the abnormal demands made on it, and also suffering from the influence of the hemolytic agent or some other toxin.

In pernicious anemia, like exophthalmic goiter, the course of the disease tends to be interrupted by periods of comparatively good health and normal bodily func. tions. Each recurrence, however, further damages the organs which are affected by the toxin, and although with the interruption of the process in its early stages there may be complete restoration to health, sooner or later, regardless of the further course of the disease, permanent injury results.

The ability of man to develop other hematopoietic centers, when the normal source of the supply of blood corpuscles, the bone marrow, is under great stress, is well known. Such centers have been demonstrated in the spleen, the liver and lymph nodes. Whether in pernicious anemia the augmentation of the hematopoietic functions is simply compensatory by reason of the increased blood destruction of the disease, or whether the bone marrow, under the influence of some specific toxin, is primarily diseased, so that immature red cells are put into the circulation, is as yet problematic. It is more than possible that pernicious anemia could be cured in its inception, like cancer, if treated properly; when it is well established, a vicious circle is produced and a permanently disabled hematopoietic system is added to the primary etiologic factor or factors. The question of permanent cure in pernicious anemia, therefore, may ultimately depend, not only on interrupting the progress of the disease by removing what appears to be the primary factor, but also to a greater extent on our ability to restore the bone marrow and other hematopoietic structures to their normal physiologic functions. It is to be hoped that earlier recognition of pernicious anemia will result in earlier splenectomy. 
Activity of the Disease:-The activity of the disease is an important consideration in discussing the advisability of operation. Splenectomy should be undertaken with hesitation in acute stages of pernicious anemia and especially with a falling blood picture. The operation cannot at the present time be advocated when cord changes and other permanent pathologic processes mark the terminal stages of the disease. Moreover, as already suggested, if it is not possible to tide a patient over the critical periods by transfusion, splenectomy is contraindicated. Definite statements based on the qualitative blood picture cannot be made, but if the hemoglobin is under 25 per cent. and cannot be raised, operative treatment is of considerable risk and has doubtful results.

The extent of the disability of the patient must of course be carefully considered. If a patient with every evidence of the disease is able to carry on his usual occupation, splenectomy cannot be strongly advocated, yet these may be the very patients in whom good results may be obtained. The operation may be urged more legitimately when the patient is unable to do his work or is a chronic invalid.

A positive diagnosis of pernicious anemia having been made and the advisability of splenectomy established, it is important that the patient and his family be correctly informed as to the purpose and the status of splenectomy.

Splenectomy we have found of less technical difficulty in pernicious anemia than in any other disease or condition. An incision toward the outer edge of the left rectus extending above to about one inch from the costal margin and below to the level of the umbilicus is satisfactory. An abdominal exploration should first be made, especially in view of the frequent complications of liver and gallbladder met with in those diseases for which splenectomy is advocated.

The dislocation of the spleen from its position against the diaphragm and against the left kidney is not, as a rule, complicated by formidable adhesions in pernicious anemia; nevertheless, the operation is facilitated and the minor oozing points are controlled by the introduction of a large abdominal pack against the newly exposed surface. In the actual removal of the spleen it must always be borne in mind that the stomach and pancreas are in close anatomic relationship. The fundus of the stomach is in such immediate apposition to the gastric surface of the spleen that its wall may be injured if the gastrosplenic omentum at this point is not divided with particular care.

The tail of the pancreas does not bear a constant relationship to the splenic pedicle. In some instances it lies in front; in others behind, and it may extend along the pedicle until its tip rests in the hilus of the spleen. It is quite evident, in certain cases, that the tip of the pancreas must be dislodged from its position if the pedicle is to be ligated without injury, although such a condition is relatively unusual in pernicious anemia. The pedicle is rarely of sufficient size to present any difficulties in its control. It is comparatively easy to isolate the artery and the veins and ligate them independently. Although it has been advised that the artery should be ligated first in order that the spleen may empty itself of its blc d, and although I have done this in some cases, I have never been able to observe any difference in the convalescence or the effect of splenectomy on these patients, as compared with those in whom the artery and veins were ligated at the same time.
All bleeding points having been securely controlled, the pack is removed and the diaphragmatic surface inspected.

\section{SUMMARY}

We believe that splenectomy should be considered in every case of pernicious anemia in which the diagnosis has been established and all possible etiologic factors which might be independently remedied have been excluded. The failure of other means to combat the disease, the previous fatal prognosis, and the low operative mortality are strong arguments in favor of splenectomy. Contraindications must be positive and adequate. Granting this, however, we must be cautioned by our imperfect knowledge of the surgical indications. The conservative selection of cases is therefore advisable.

\section{PERNICIOUS ANEMIA TREATED BY SPLE- NECTOMY AND SYSTEMATIC, OFTEN- REPEATED TRANSFUSION OF BLOOD}

TRANSFUSION IN BENZOL PCISONING *

ROY D. MCCLURE, M.D. DETROIT

Transfusion of blood in cases of pernicious anemia is perhaps the first remedy that suggests itself for the treatment of the malady. It probably was tried long before it is recorded in the literature. Since the newer ways of transfusion with syringes and the citrate method have so simplified the operation, it has been tried innumerable times, but with only indifferent success. The best it has accomplished has been to initiate a remission in the course of the disease. There are many reasons why these transfusions have not been of more value. Chief among these is the lack of systematic treatment. Usually about as much good is accomplished by a single transfusion as is accomplished by a single inunction of mercury in the treatment of syphilis. Discredit has also been incurred by using blood which has not stood the proper tests and which is not only unsuitable but is often positively harmful and which may result in disaster.

The practice of transfusion of blood since it was first performed in man in 1666 by Jeane Baptise Denys, professor of philosophy and mathematics in the Royal University in Paris and physician to Louis XIV, and by his teacher Dr. Lower ${ }^{1}$ in London, who had developed the method by his work with his experiments on animals in 1660, has passed through periods of great disrepute. At first it fell into disfavor because the blood of animals, dogs, sheep and calves was used and there were probably deaths from anaphylaxis. The road to safe transfusion was not made possible until Landois ${ }^{2}$ in Greifswald in 1870 discovered that the blood of one man was not always compatible with the blood of others, though I believe he never explained it, yet he did show that the serum of one animal might dissolve the red blood cells of another animal. It remained for Moss $^{3}$ in this coun-

\footnotetext{
* From the Surgical Clinic of Dr. W. S. Halsted, Johns Hopkins Hospital.

Read before the joint meeting of the Section on Practice of Medicine and the Section on Surgery, General and Abdominal, at the Sixty-Seventh Annual Session of the American Medical Association, Detroit, 1916.

1. Philosophical Transactions and Collections of Medical and Philog ical Papers, John Lowthrop, 1731

2. Landois: Die Transfusion des Blutes, 1875

3. Moss: Bull. Johns Hopkins Hosp., 1910, xxi, 63.
} 